Please do not remove this page

RMIT

UNIVERSITY

\title{
Next generation flight management system for real-time trajectory based operations
}

Ramasamy, Subramanian; Sabatini, Roberto; Gardi, Alessandro; Kistan, Trevor

https://researchrepository.rmit.edu.au/esploro/outputs/9921862186801341/filesAndLinks?institution=61RMIT_INST\&index=null

Ramasamy, S., Sabatini, R., Gardi, A., \& Kistan, T. (2014). Next generation flight management system for real-time trajectory based operations. Applied Mechanics and Materials, 629, 344-349.

https://doi.org/10.4028/www.scientific.net/AMM.629.344

Document Version: Accepted Manuscript

Published Version: https://doi.org/10.4028/www.scientific.net/AMM.629.344

Repository homepage: https://researchrepository.rmit.edu.au

(C) 2014 Applied Mechanics and Materials

Downloaded On 2023/04/26 13:42:36 +1000

Please do not remove this page 
Thank you for downloading this document from the RMIT Research Repository.

The RMIT Research Repository is an open access database showcasing the research outputs of RMIT University researchers.

RMIT Research Repository: http://researchbank.rmit.edu.au/

\section{Citation:}

Ramasamy, S, Sabatini, R, Gardi, A and Kistan, T 2014, 'Next generation flight management system for real-time trajectory based operations', Applied Mechanics and Materials, vol. 629, pp. 344-349.

See this record in the RMIT Research Repository at:

https://researchbank.rmit.edu.au/view/rmit:24972

Version: Accepted Manuscript

Copyright Statement: (c) 2014 Applied Mechanics and Materials

Link to Published Version:

http://dx.doi.org/10.4028/www.scientific.net/AMM.629.344 


\title{
Next Generation Flight Management System for Real-Time Trajectory Based Operations
}

\section{Subramanian Ramasamy ${ }^{1}$, Roberto Sabatini ${ }^{2, a^{*}}$, Alessandro Gardi ${ }^{3}$ and Trevor Kistan ${ }^{4}$}

\author{
1,2,3,4 School of Aerospace, Mechanical and Manufacturing Engineering \\ RMIT University, Melbourne, VIC 3000, Australia \\ aroberto.sabatini@rmit.edu.au
}

\begin{abstract}
Keywords: flight management system, four dimensional trajectory optimisation, intent based operation, stochastic trajectory
\end{abstract}

\begin{abstract}
This paper presents the concept of operations, architecture and trajectory optimisation algorithms of a Next Generation Flight Management System (NG-FMS). The NG-FMS is developed for Four Dimensional (4D) Intent Based Operations (IBO) in the next generation Communications, Navigation, Surveillance and Air Traffic Management system (CNS+A) context. The NG-FMS, primarily responsible for the aircraft navigation and guidance task, acts as a key enabler for achieving higher level of operational efficiency and mitigating environmental impacts both in manned and unmanned aircraft applications. The NG-FMS is interoperable with the future ground based 4DT Planning, Negotiation and Validation (4-PNV) systems, enabling automated Trajectory/Intent Based Operations (TBO/IBO). After the NG-FMS architecture is presented, the key mathematical models describing the trajectory generation and optimisation modes are introduced. A detailed error analysis is performed and the uncertainties affecting the nominal trajectories are studied to obtain the total NG-FMS error budgets. These are compared with the Required Navigation Performance (RNP) values for the various operational flight tasks considered.
\end{abstract}

\section{Introduction}

Conventionally, the Flight Management System (FMS) acts as the key enabler of automated navigation and guidance services in manned aircraft. The FMS main goal is to reduce the pilot's workload by acting both as a mission planner and a mission monitor. However, there has been a rapid expansion of global air transport and at the same time; a growing concern for environmental consciousness has resulted in active research on improving operational efficiency and safety, while reducing the environmental impacts of aviation [1, 2]. In the Communication, Navigation, Surveillance, Air Traffic Management (CNS/ATM) and Avionics (CNS+A) domain, novel technologies have to be developed to achieve these fundamental goals. In this context, the Next Generation Flight Management System (NG-FMS) is a key enabler for generating globally optimal trajectories that fulfil the evolving operational, safety and environmental requirements. The NGFMS is developed for Four Dimensional (4D) Trajectory/Intent Based Operations (TBO/IBO) in combination with the Next Generation Air Traffic Management (NG-ATM) systems and Next Generation Airborne Data Link (NG-ADL) communications. The efficiency and effectiveness of NG-ATM strategies are directly driven by the nature of information sharing and its underlying operational and technological frameworks. In the recent years, Unmanned Aircraft (UA) are increasingly used for a number of applications and the need for their integration into the civilian airspace has led to the development of a host of dedicated automation services. In this perspective, cooperative and non-cooperative Sense-and-Avoid (SAA) systems are key technology enablers that can support the UA to access non-segregated airspace and hence they are incorporated as part of the NG-FMS. Additionally, suitable processing/interfaces required for achieving Performance Based Operations $(\mathrm{PBO})$ are considered essential requirements to be addressed as part of the $\mathrm{CNS}+\mathrm{A}$ system design. 


\section{NG-FMS Architecture}

The NG-FMS architecture is primarily based on the core functionalities namely flight planning (FPLN), localisation and state determination, trajectory optimisation (TRAJ), performance predictions (PRED) and guidance. Additionally, the FMS also provides auto-throttle controls for engines and communicates with the 4-PNV system. The NG-FMS core is based on a multiobjective and multi-model 4D-Trajectory (4DT) optimisation approach. The databases associated are Magnetic Deviation Database (MAG-DB), Navigation Database (NAV-DB) and aircraft Performance Database (PERF-DB). The NG-FMS is also termed as Next Generation Mission Management System (NG-MMS) incorporating UA mission planning tasks. The NG-FMS detailed architecture is illustrated in Fig. 1. To accomplish the above mentioned tasks, the primary NG-FMS modules are:

- Trajectory Planning/ Optimisation - This module performs 4DT planning and optimisation functions for pre-tactical, tactical and emergency situations. The 4DT optimiser includes the models pool and constraints pool involves a number of cost functions.

- Trajectory Monitoring - It performs state estimation, calculating the deviations between the active 4DT intents and the estimated/predicted aircraft states.

- Path Correction - It corrects the path deviation in terms of lateral, vertical and time profiles and the generated steering commands are provided to the guidance module of the NG-FMS.

- Trajectory Negotiation and Validation - It carries out the process of negotiation, which can be initiated by the pilot via the NG-FMS or by the 4-PNV system.

- FMS Performance Manager - It monitors the active 4DT intents for errors to address integrity requirements. The integrity analysis module is based on RNP, Required Communication Performance (RCP) and Required Surveillance Performance (RSP) managers.

- FMS Integrity Manager - This module is based on Avionics-Based Integrity Augmentation (ABIA) [3-5]. The inputs from the different sensor candidates are augmented based on predefined decision logics and the result is passed as input to an Integrity Flag Generator (IFG).

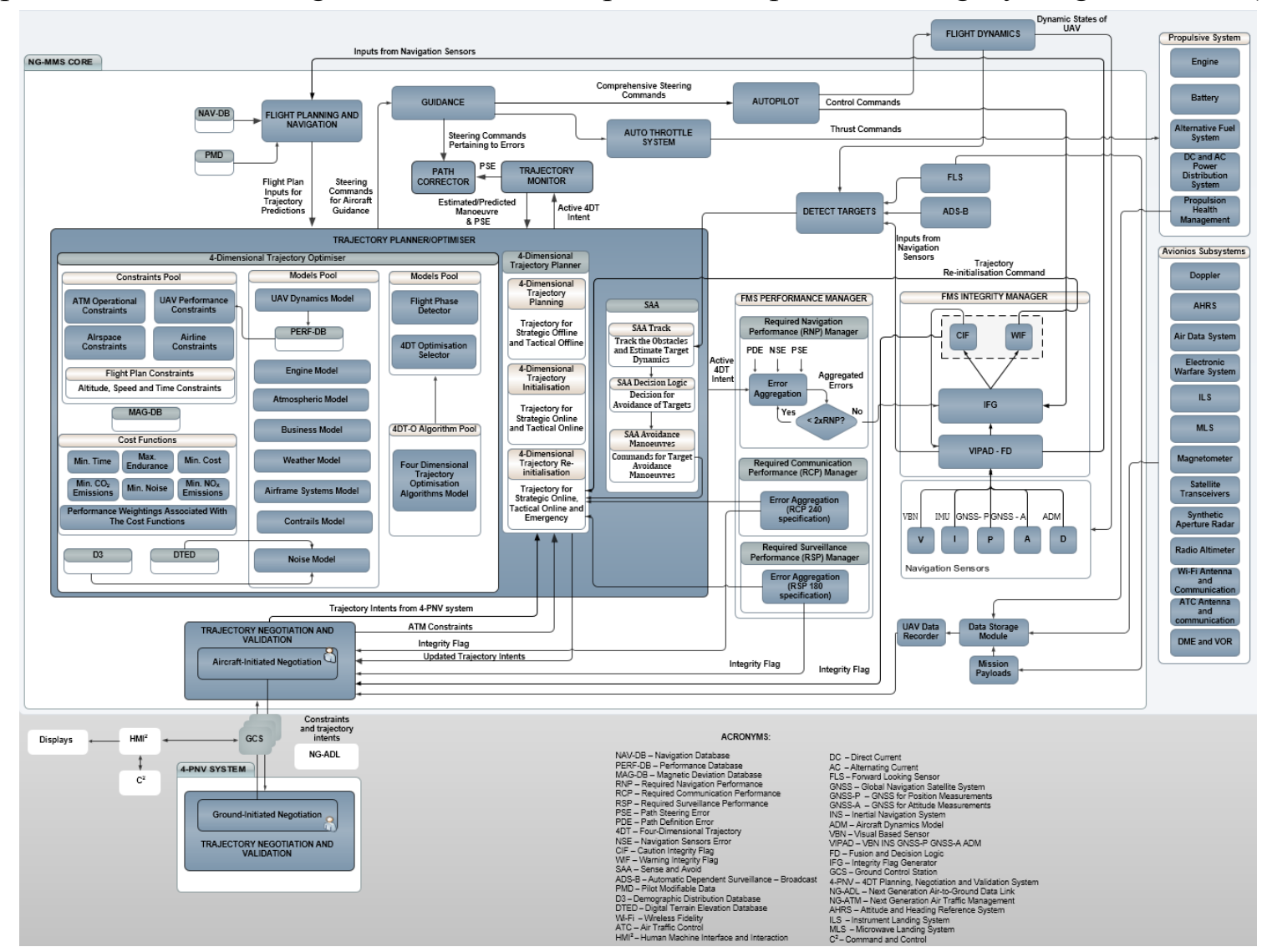

Fig. 1. NG-FMS architecture. 


\section{Considerations for datalink mediated NG-FMS and 4-PNV system interoperability}

Aircraft Communications Addressing and Reporting System (ACARS) mediated Future Air Navigation System (FANS) 1/A Controller-Pilot Data Link Communications (CPDLC) messages are currently used to communicate constraints known to the ground system to the airborne FMS. The key requirement in the implementation of IBO is the exchange of actual trajectory information between the NG-FMS and the 4-PNV system. This is feasible with current FANS-1/A technology [6], but the communicated route changes should be kept minimal and localised around the actual deviation, with that portion of the route that remains unaltered after the deviation returns to the original route not being communicated. Since the goal is to achieve optimisation over the strategic and tactical online phases of the flight and not only deviations around local airspace restrictions, a more general mechanism is required. The bandwidth requirements and the constraints are taken into account. The use of a reduced alphabet to communicate trajectory information between the 4-PNV system and large numbers of NG-FMS systems may be a practical necessity even if the computed optimisations are degraded. This could be attempted in the context of a mixed centraliseddecentralised separation management system that has some similarities to the collaborative processing performed by the 4-PNV and NG-FMS in concert.

\section{Mathematical Models}

The NG-FMS generates 4DT intents composed by groups of single trajectories $t$ belonging to the global set $T$. There are $n$ waypoints in each generated trajectory and these are denoted as $\left\{\boldsymbol{w}_{1}^{t}, \boldsymbol{w}_{2}^{t} \ldots \boldsymbol{w}_{\boldsymbol{n}}^{t}\right\}$. The successive waypoints of the trajectory are defined with respect to the previous waypoints by conditional probability and generate fly-by and fly-over waypoints for each flight segment until the destination is reached. The NG-FMS trajectory optimisation algorithms are based on a 3-degree-of-freedom (3-DoF) point mass Aircraft Dynamics Model (ADM) with variable mass [7]. The 3-DoF equations of motion describing the aircraft states and governing the translational movements along the longitudinal, lateral and vertical axes are:

$$
\begin{aligned}
\frac{d \varphi}{d t} & =\frac{V \cos \gamma \sin \chi+V_{W_{\varphi}}}{R_{M}+h} \\
\frac{d \lambda}{d t} & =\frac{V \cos \gamma \cos \chi+V_{W_{\lambda}}}{\cos \varphi\left(R_{T}+h\right)} \\
\frac{d h}{d t} & =V \sin \gamma+V_{W_{h}} \\
\frac{d V}{d t} & =\frac{\Delta f}{m}-g \sin \gamma \\
\frac{d \gamma}{d t} & =\frac{g(n \cos \phi-\cos \gamma)}{V} \\
\frac{d \chi}{d t} & =\left(\frac{N \cdot g}{V}\right)\left(\frac{\sin \phi}{\cos \gamma}\right) \\
\frac{d m}{d t} & =-c(P, V, h) T(P, V, h) \\
\Delta f & =T(P, V, h)-D(L, V, h)
\end{aligned}
$$

The above Differential Algebraic Equations (DAEs) incorporate three control variables $u=(P, n, \phi)$ where $P$ is the engine power setting, $n$ is the load factor and $\phi$ is the bank angle. These form the inputs of the dynamic system. The seven state variables are described as $x=(m, \varphi, \lambda, h, V, \gamma, \chi)$, the derivatives of which are presented in the EOM, where $m$ is the aircraft mass, $\varphi$ is the geodetic latitude, $\lambda$ is the geodetic longitude, $h$ is the altitude, $V$ is the true air speed, $\gamma$ is the flight path angle, $\chi$ is the heading, $R_{M}$ is the meridional radius of curvature, $R_{T}$ is the transverse radius of curvature, $W$ is the wind velocity and $g$ is the acceleration due to gravity of the Earth. Assumptions considered are a rigid body aircraft, nil wing bending effect, rigidly mounted aircraft engine on the vehicle body, zero thrust angle, the location of the aircraft mass in the aircraft centre of gravity, varying mass only as a result of fuel consumption, no sideslip and uniform 
gravity. Wind effects are considered along the three translational axes of the 3-DOF EOM. The geodetic coordinate reference system used is the World Geodetic System of year 1984 (WGS 84). The optimisation problem of determining the states $x(t)$ depends on the performance index, $J$ denoted by a sum of Mayer $(\Phi)$ and Lagrange $(L)$ terms and it is given by:

$$
J=\Phi\left[x\left(t_{0}\right), x\left(t_{f}\right), t\right]+\int_{t_{0}}^{t_{f}} L[x(t), u(t), t] d t
$$

where $t_{0}$ is the initial time and $t_{f}$ is the final time in the time epoch, $t$. The dynamic constraints defining the derivatives of the state vector are given by:

$$
\dot{x}(t)=f[x(t), u(t), t]
$$

The path constraints are expressed as:

$$
C_{\min } \leq C[x(t), u(t), t ; p] \leq C_{\max }
$$

The boundary conditions describing the initial and final states are given by:

$$
\Phi_{\min } \leq \Phi\left[x\left(t_{0}\right), x\left(t_{f}\right), u\left(t_{0}\right), u\left(t_{f}\right) ; p\right] \leq \Phi_{\max }
$$

Error modelling is performed to determine the effects of uncertainties on the 4D trajectories generated. The errors might be due to database accuracy degradations, system modelling errors, atmospheric disturbances and subsystem errors [7]. The random errors, which are unpredictable, are quantified to estimate the overall error associated with the position of the aircraft. The system states are modified with the addition of the stochastic term, $e(t)$ and are given by:

$$
\dot{x}(t)=f[x(t), u(t), e(t), t]
$$

The errors associated with the position of the aircraft (both manned and unmanned) are dependent on the error deviations of the ADM parameters given by:

$$
\begin{aligned}
& \sigma_{\dot{V}}=\sqrt{(-g \cos \gamma)^{2} \sigma_{\gamma}^{2}+\left(\frac{\text { Tnorm }}{m}\right)^{2} \sigma_{\tau}^{2}} \\
& \sigma_{\dot{h}}=\sqrt{(\sin \gamma)^{2} \sigma_{V}^{2}+{\sigma_{V_{W_{h}}}}^{2}+(V \cos \gamma)^{2}{\sigma_{\gamma}}^{2}} \\
& \sigma_{\dot{\gamma}}=\sqrt{\left[\frac{g(-N \cdot \cos \phi+\cos \gamma)}{V^{2}}\right]^{2} \sigma_{V}^{2}+\left(-\frac{N \cdot g \cdot \sin \phi}{V}\right)^{2} \sigma_{\phi}^{2}+\left(-\frac{g \cdot \sin \gamma}{V^{2}}\right)^{2} \sigma_{\gamma}^{2}} \\
& \sigma_{\dot{\chi}}=\sqrt{\left(\frac{-N \cdot g \cdot \sin \phi}{\cos \gamma \cdot V^{2}}\right)^{2} \sigma_{V}^{2}+\left(\frac{N \cdot g \cdot \cos \phi}{V \cdot \cos \gamma}\right)^{2} \sigma_{\phi}{ }^{2}+\left(\frac{N \cdot g \cdot \sin \phi \cdot \sin \gamma}{\gamma \cdot \cos \gamma^{2}}\right)^{2} \sigma_{\gamma}{ }^{2}} \\
& \sigma_{\dot{\varphi}}=\sqrt{\frac{\left[\frac{1}{\left(R_{M}+h\right)}\right]^{2} \sigma_{V_{W_{\varphi}}}^{2}+\left[\frac{-V \cdot \sin \gamma \cdot \sin \chi+V_{W_{\varphi}}}{\left(R_{M}+h\right)^{2}}\right]^{2} \sigma_{h}{ }^{2}+\left[\frac{-V \cdot \sin \gamma \cdot \sin \chi}{\left(R_{M}+h\right)}\right]^{2} \sigma_{\gamma}{ }^{2}+\cdots}{\left[\frac{\cos \gamma \cdot \sin \phi}{\left(R_{M}+h\right)}\right]^{2} \sigma_{V}{ }^{2}+\left[\frac{V \cdot \sin \gamma \cdot \cos \chi}{\left(R_{M}+h\right)}\right]^{2} \sigma_{\chi}^{2}}} \\
& \sigma_{\dot{\lambda}}=\sqrt{\begin{array}{c}
{\left[\frac{\cos \gamma \cdot \cos \chi}{\cos \varphi\left(R_{T}+h\right)}\right]^{2} \sigma_{V}{ }^{2}+\left[\frac{-V \cdot \cos \gamma \cdot \sin \chi}{\cos \varphi\left(R_{T}+h\right)}\right]^{2} \sigma_{\chi}{ }^{2}+\left[\frac{\sin \varphi \cdot \cos \varphi\left(R_{T}+h\right)\left(V \cdot \cos \gamma \cdot \cos \chi+V_{\left.W_{\lambda}\right)}\right.}{\left(\cos \varphi\left(R_{T}+h\right)\right)^{2}}\right]^{2} \sigma_{\varphi}{ }^{2}+\cdots} \\
{\left[\frac{1}{\cos \varphi\left(R_{T}+h\right)}\right]^{2}{\sigma_{V_{W_{\lambda}}}}^{2}+\left[\frac{-\cos \varphi\left(V \cdot \sin \gamma \cdot \cos \chi+V_{\left.W_{\lambda}\right)}\right.}{\left(\cos \varphi\left(R_{T}+h\right)\right)^{2}}\right]^{2} \sigma_{h}{ }^{2}+\left[\frac{-V \cdot \sin \gamma \cdot \cos \chi}{\cos \varphi\left(R_{T}+h\right)}\right]^{2} \sigma_{\gamma}{ }^{2}}
\end{array}}
\end{aligned}
$$

The errors in aircraft positioning are conveniently described by a navigation error ellipsoid. For cooperative and non-cooperative obstacle avoidance and safe-separation maintenance, the overall uncertainty volume is obtained by combining the navigation error ellipsoid with the tracking error ellipsoid and then translating them to unified range and bearing uncertainty descriptors [8].

\section{NG-FMS Simulation}

A simulation case study was accomplished using an Airbus A380 aircraft with maximum take-

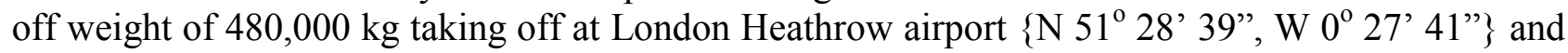
landing at Singapore Changi airport $\left\{{\mathrm{N} 01^{\circ}}^{\circ} 21^{\prime} 33.16 ”, \mathrm{E} 103^{\circ} 59^{\prime} 21.57^{\prime}\right\}$. The aircraft parameters and aerodynamic data were extracted from the EUROCONTROL Base of Aircraft Data (BADA) 
[9]. Objectives such as fuel consumption, time and path constraints are considered via an appropriate set of performance weightings agreed between the Air Navigation Service Provider (ANSP) and the Airline Operation Centre (AOC). Depending on the cost functions and gains selected (i.e., minimum time, fuel and other environmental costs such as gaseous emissions of $\mathrm{CO}_{2}$, $\mathrm{NO}_{\mathrm{x}}$, etc.), there are different trajectory possibilities for all the flight segments. Fig. 2 illustrates the different trajectories generated, each resulting from a different cost function on time and fuel minimisation. For the climb phase, the maximum $\mathrm{CO}_{2}$ and $\mathrm{NO}_{\mathrm{x}}$ reductions are $281.7 \mathrm{~kg}$ and $1.5 \mathrm{~kg}$ respectively. The trajectory corresponding to minimum fuel burn provides $90 \mathrm{~kg}$ fuel savings when compared to the minimum time optimisation case. These results are tabulated in Table 1.

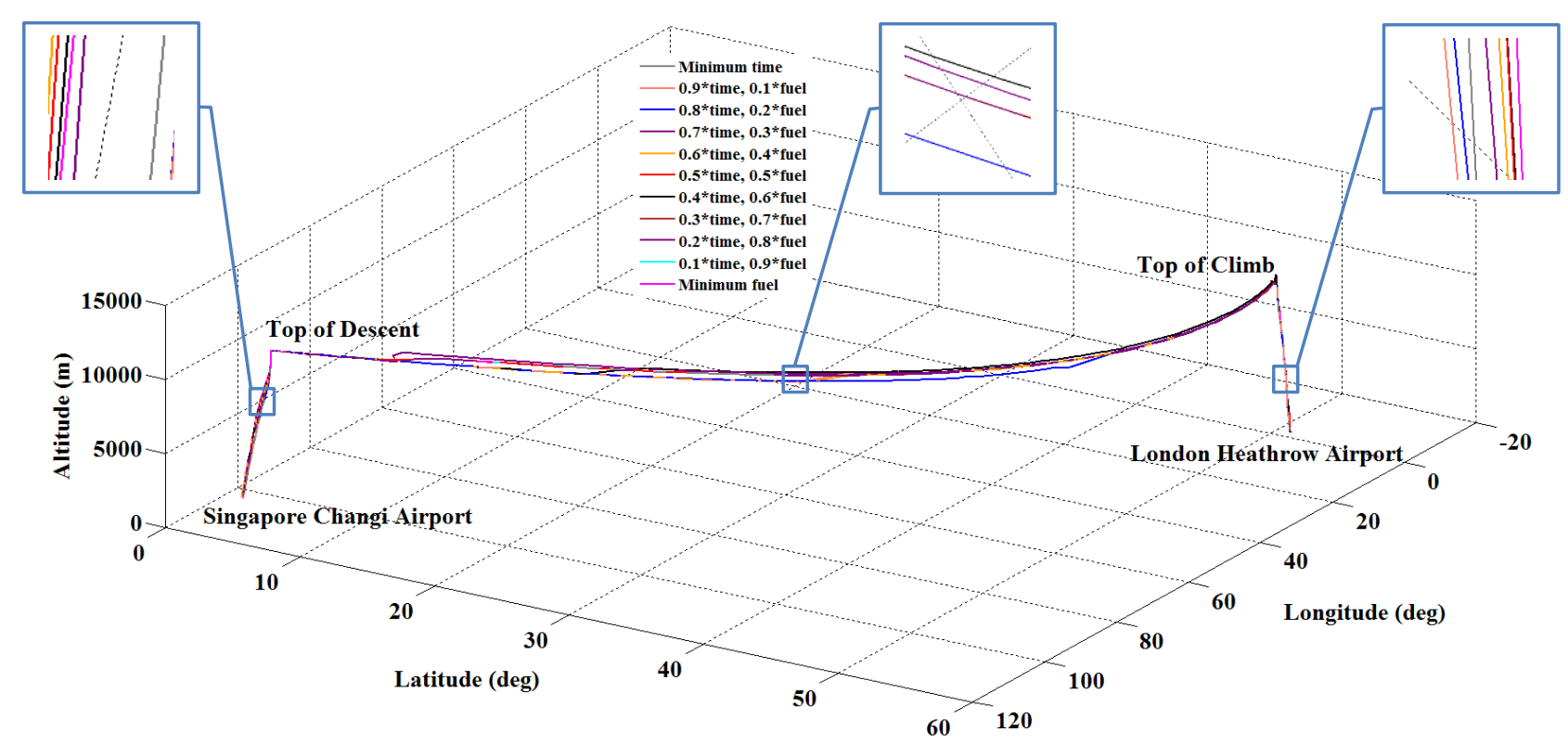

Figure 2. 4D trajectories.

Table 1. Time, fuel burn and emissions of the 4D trajectories.

\begin{tabular}{|c|c|c|c|c|}
\hline Reference trajectory & Time $[\mathrm{sec}]$ & Fuel $[\mathrm{kg}]$ & $\mathrm{CO}_{2}[\mathrm{~kg}]$ & $\mathrm{NO}_{\mathrm{x}}[\mathrm{kg}]$ \\
\hline Minimum time & 572 & 5010.0 & 15681.3 & 80.7 \\
\hline $0.9 *$ time, $0.1 *$ fuel & 574 & 5000.0 & 15650.0 & 80.5 \\
\hline $0.8 *$ time, $0.2 *$ fuel & 577 & 4990.0 & 15618.5 & 80.2 \\
\hline $0.7 *$ time, $0.3 *$ fuel & 580 & 4980.0 & 15587.4 & 80.1 \\
\hline $0.6 *$ time, $0.4 *$ fuel & 582 & 4971.0 & 15559.2 & 80.0 \\
\hline $0.5 *$ time, $0.5 *$ fuel & 584 & 4960.0 & 15524.8 & 79.9 \\
\hline $0.4 *$ time, $0.6 *$ fuel & 585 & 4954.0 & 15506.0 & 79.8 \\
\hline $0.3 *$ time, $0.7 *$ fuel & 586 & 4945.0 & 15477.8 & 79.6 \\
\hline $0.2 *$ time, $0.8 *$ fuel & 587 & 4937.0 & 15452.8 & 79.5 \\
\hline $0.1 *$ time, $0.9 *$ fuel & 588 & 4928.0 & 15424.6 & 79.3 \\
\hline Minimum fuel & 589 & 4920.0 & 15399.6 & 79.2 \\
\hline
\end{tabular}

A stochastic analysis case study was performed to evaluate the potential impact of system uncertainties on the trajectory generation process. The introduction of uncertainties on all nominal parameters, with ranges equal to the standard deviations as provided in [10] allows transforming the EOM into stochastic differential equations as given in Eq. 14 to Eq. 19 that are then treated with the Monte Carlo sampling technique and solved using the deterministic optimizer for 100 samples. Considering these results for the climb phase, the two-sigma confidence region, corresponding to $1.85 \mathrm{NM}$ falls well inside the RNP requirements of $2 \mathrm{NM}$. 


\section{Conclusions and Future Work}

The trajectory optimisation algorithms of the NG-FMS were presented, providing 4-Dimensional Trajectory Optimisation (4DT-O) and air-to-ground trajectory negotiation/validation functionalities interoperable with future ATM systems [11] to support intent based operations. A detailed functional architecture of the NG-FMS suitable for both manned and unmanned aircraft was described. The key mathematical models developed to implement the 4DT-O capability, addressing both the deterministic and stochastic trajectory optimisation problems were presented. Investigation of the total NG-FMS system error boundaries was conducted using the Monte Carlo sampling technique to evaluate the effects of uncertainties propagation. Realistic simulation case studies were performed and the results demonstrated the functional capability of the NG-FMS to generate costeffective trajectory profiles satisfying the operational and environmental constraints. Additionally, the NG-FMS trajectory error budget was obtained and compared with the applicable RNP thresholds demonstrating compliance at the 2-sigma level. Future research will address the stochastic analysis with other distributions and will incorporate a number of integrated navigation architectures. Additionally, Avionics-Based Integrity Augmentation architecture [3-5] will be incorporated as an integral part of the CNS parameters for both manned and unmanned aircraft for varied applications [12].

\section{References}

[1] D. Koehl, The Updated of the European ATM Master Plan, SESAR, Amsterdam. (2012)

[2] H. Erzberger, Optimum Climb and Descent Trajectories for Airline Mission, Advisory Group for Aerospace Research and Development (AGARD), 251(9), pp. 1-15 (1981)

[3] R. Sabatini, T. Moore and C. Hill, A new avionics-based GNSS integrity augmentation system: Part 1 - Fundamentals, Journal of Navigation, 66(3), pp. 363-384. (2013) DOI: $10.1017 / \mathrm{S} 0373463313000027$

[4] R. Sabatini, T. Moore and C. Hill, A new avionics-based GNSS integrity augmentation system: Part 2 - Integrity flags, Journal of Navigation, 66(4), pp. 501-522. (2013) DOI: $10.1017 / \mathrm{S} 0373463313000143$

[5] R. Sabatini, T. Moore and C. Hill, Avionics-Based Integrity Augmentation System for Missionand Safety-Critical GNSS Applications, $25^{\text {th }}$ International Technical Meeting of The Satellite Division of the Institute of Navigation - ION GNSS, Tennessee, USA. (2012)

[6] E. Mueller, Experimental Evaluation of an Integrated Datalink and Automation-Based Strategic Trajectory Concept, $7^{\text {th }}$ AIAA Aviation Technology, Integration and Operations Conference (ATIO), Belfast. (2007) DOI: 10.2514/6.2007-7777

[7] S. Ramasamy, R. Sabatini, A. Gardi and Y. Liu, Novel flight management system for real-time 4-dimensional trajectory based operations, AIAA Guidance, Navigation, and Control Conference 2013 (GNC2013), Boston, MA, USA (2013) DOI: 10.2514/6.2013-4763

[8] S. Ramasamy, R. Sabatini and A. Gardi, Avionics Sensor Fusion for Small Size Unmanned Aircraft Sense-and-Avoid, IEEE Metrology for Aerospace Conference, Benevento, Italy. (2014)

[9] User Manual for the Base of Aircraft Data (BADA), Revision 3.8, EUROCONTROL. (2000)

[10] R. Sabatini, C. Bartel, A. Kaharkar and T. Shaid, Low-cost Vision Sensors and Integrated Systems for Unmanned Aerial Vehicle Navigation and Guidance, ARPN Journal of Systems and Software, 2(11), pp. 323-349. (2012)

[11] A. Gardi, R. Sabatini, S. Ramasamy and K. de Ridder, 4-Dimensional Trajectory Negotiation and Validation System for the Next Generation Air Traffic Management, AIAA Guidance, Navigation, and Control Conference 2013 (GNC2013), Boston, MA, USA (2013) DOI: $10.2514 / 6.2013-4893$

[12] A. Gardi and R. Sabatini, Unmanned Aircraft Bistatic Lidar for $\mathrm{CO}_{2}$ Colum Density Determination, IEEE Metrology for Aerospace Conference 2014, Benevento, Italy. (2014) 